\title{
Gasto farmacéutico en diabetes mellitus en una región de España según el Clinical Risk Group, 2012
}

\section{Pharmaceutical Expenditure for Diabetes Mellitus in a Region of Spain as Clinical Risk Group, 2012}

\section{Despesa com medicamentos em pacientes com diabetes mellitus em uma região da Espanha como o Grupo de Risco Clínico de 2012}

Fecha de recepción: 16-03-15 Fecha de aceptación: 18-02-16 Disponible en línea: 03-05-16 doi:10.11144/Javeriana.rgyps15-30.gfdm

Cómo citar este artículo:

Alvis-Estrada L, Vivas-Consuelo D, Caballer-Tarazona Vicent, Usó-Talamantes R, Sancho-Mestre C, Buigues-Pastor L. Gasto farmacéutico en diabetes mellitus en una región de España según el Clinical Risk Group, 2012. Rev. Gerenc. Polít. Salud. 2016; 15(30): 68-78. http://dx.doi.org/10.11144/Javeriana. rgyps15-30.gfdm

Luis Alvis-Estrada*

David Vivas-Consuelo**

Vicent Caballer-Tarazona***

Ruth Usó-Talamantes $* * * *$

Carla Sancho-Mestre $* * * * *$

Laia Buigues-Pastor******

Economista, miembro del Grupo de Investigación en Economía de la Salud de la Universidad de Cartagena. Campus de la Salud, Zaragocilla, Facultad de Enfermería. Correo electrónico: lalvis20@yahoo.com

** Médico, profesor de la Universitat Politècnica de València, España, Facultad de Administración y Dirección de Empresas, director del Centro de Ingeniería Económica y Gestión de la Salud. Correo electrónico: dvivas@upvnet.upv.es

*** Licenciado en Administración, Universitat Politècnica de València, España, Facultad de Administración y Dirección de Empresas. Miembro del Centro de Ingeniería Económica y Gestión de la Salud. Correo electrónico: vincabta@ hotmail.com

***** Médico, Conselleria de Sanitat, Comunidad Valenciana, España. Correo electrónico: uso_rut@gva.es

$68{ }^{* * * * *}$ Economista, Universitat Politècnica de València, España, Centro de Investigación en Economía y Gestión de la Salud. Correo electrónico: carsan13@ade.upv.es

******** Licenciada en Economía. Conselleria de Sanitat, Comunidad Valenciana, España. Correo electrónico: buigues_lai@gva.es 


\section{Resumen}

Se pretende estimar la multimorbilidad asociada con diabetes mellitus tipo 2 y su relación con el gasto farmacéutico, para lo cual se realizó un estudio de corte transversal durante el año 2012. Se identificó a 350015 individuos diabéticos, a través de códigos clínicos, usando la Clasificación Internacional de Enfermedades y el software 3M Clinical Risk Groups. Todos los pacientes fueron clasificados en cuatro grupos de morbilidad. El primer grupo corresponde al estadio inicial, el segundo grupo incluye el núcleo de multimorbilidad de pacientes en fases intermedia y avanzada, el tercer grupo incluye pacientes con diabetes y enfermedades malignas, y el último grupo es de pacientes en estado catastrófico, principalmente enfermos renales crónicos. La prevalencia bruta de diabetes fue de 6,7\%. El gasto promedio total fue de $€ 1257,1$. La diabetes se caracteriza por una fuerte presencia de otras condiciones crónicas y tiene un gran impacto en el gasto farmacéutico.

Palabras clave: diabetes mellitus tipo 2; costos de los medicamentos; ajuste de riesgo; hipoglucemiantes; enfermedad crónica; comorbilidad

\section{Abstract}

Estimations of multimorbidity associated with Type 2 Diabetes Mellitus and its relationship to pharmaceutical expenditure. Cross-sectional study during 2012. 350,015 diabetic individuals, identified through clinical codes using the International Statistical Classification of Diseases and Related Health Problem and the 3M Clinical Risk Groups software. The raw prevalence of diabetes was $6.7 \%$. All patients were stratified into four morbidity groups. The first group corresponds to the initial state; the second group includes the core multimorbidity patients in the intermediate and advanced stages; the third group includes patients with diabetes and malignancies; the last group patients with catastrophic statuses, manly chronic renal patients. The raw prevalence of diabetes was $6.7 \%$. The average total cost was $€ 1257.1$. Diabetes is characterized by a strong presence of other chronic conditions have a great impact on pharmaceutical spending.

Keywords: type 2 diabetes mellitus; drug costs; risk adjustment; hypoglycemic agents; chronic diseases; comorbidity

\section{Resumo}

As estimativas de vários morbidade associada com diabetes mellitus tipo 2 e sua relação com a despesa farmacêutica, para o qual um estudo transversal foi realizado em 2012. Ele foi identificado em 350015 indivíduos diabéticos, foram identificados através códigos clínicos, utilizando a Classificação Internacional de Doenças e Risco clínica software Grupos 3M. Todos os pacientes foram classificados em quatro grupos de doença 4 . O primeiro grupo corresponde à fase inicial (CRG 1-4); O segundo grupo inclui pacientes multimorbid principais fases intermediárias e avançadas, o terceiro grupo inclui pacientes com diabetes e doenças malignas, eo último grupo de pacientes em estado catastrófico, pacientes renais crônicos, principalmente. A prevalência global de diabetes foi de $6,7 \%$. A despesa média total foi de $€ 1257,1$. Diabetes que se caracteriza por uma forte presença de outras condições crônicas e tieniendo um grande impacto sobre os gastos farmacêutica.

Palavras-chave: diabetes mellitus tipo 2; custos de los medicamentos; risco ajustado; hipoglicemiantes; doença crônica; comorbidade 


\section{Introducción}

La diabetes es considerada por la Organización Mundial de la Salud (OMS) una epidemia y es una de las enfermedades crónicas no transmisibles de mayor impacto socioeconómico (1). En España la prevalencia de diabetes se estima alrededor del 10\% (2), en tanto que en la Comunidad Valenciana oscila entre 35 y 41,5\%, según criterios establecidos por el National Cholesterol Educational Program Adult Treatment Panel III (NCEP ATPIII) y la Federación Internacional de Diabetes (FDI), respectivamente, siendo mayor a la de muchas regiones de España (3).

A nivel mundial, los gastos sanitarios generados por diabetes representaron el $11 \%$ del gasto total sanitario en el año 2011 (4), mientras que en España representan el $8,2 \%$ del gasto sanitario total, estimándose los costos farmacológicos en 2232 millones de euros (5). En Europa, el estudio costes de la Diabetes en Europa-Tipo 2 (CODE-2) reveló que el costo de tratar a más de diez millones de pacientes con diabetes tipo 2 en los países estudiados fue aproximadamente de US\$ 29000 millones, que representa un promedio de $5 \%$ del gasto total de atención de salud en cada país. Solo los costos por hospitalización, que incluyen el tratamiento de las complicaciones a largo plazo, como la cardiopatía, representan entre 30 y $65 \%$ de los costos globales de esta enfermedad (6).

El incremento de la expectativa de vida y el aumento de la sobrevida de enfermedades crónicas como la diabetes han traído como consecuencia el incremento de los gastos en atención en salud. En tal sentido, es pertinente que los sistemas de salud que se proponen optimizar la gestión de riesgo cuenten con un mapa estructurado de la población atendida o asegurada, con el fin de identificar grupos de riesgo al tiempo que permita monitorear los recursos invertidos.
Los sistemas de ajustes de riesgos en salud mayormente conocidos en el mundo son: 1 ) grupos de costos basados en diagnósticos (DCG), desarrollados por Ellis (7); 2) grupos clínicos ajustados (ACG), desarrollados por Starfield (8); y 3 ) los grupos de riesgo clínico (CRG), desarrollados por Hughes (9).

La implementación de estos sistemas de ajuste depende de la información de la cual se disponga, y esta puede hacer referencia al diagnóstico de los pacientes o al consumo de sustancias farmacéuticas (10).

Los CRG $(9,11)$ constituyen un sistema relativamente nuevo que clasifica a los individuos en categorías mutuamente excluyentes, utilizando los datos de las consultas o contactos médicos. Asignan cada paciente a un nivel de gravedad en función de sus condiciones crónicas de salud. Clasifican al individuo y todos los servicios médicos utilizados durante un periodo determinado de tiempo. Existen 1079 CRG básicos que se agrupan en tres niveles, a los que se hace referencia como grupos de riesgo clínico (ACRG). Los tres niveles sucesivos de agregación se denominan ACRG1 (416 grupos), ACRG2 (151 grupos) y ACRG3 (38 grupos), siendo ACRG3 el más alto nivel de agregación, que consta de nueve estados de salud.

Todos los grupos se pueden clasificar en nueve estados de salud, que mantienen la significación clínica y tienen en cuenta las necesidades futuras de atención médica y la similitud clínica de los individuos asignados a los CRG agregados. Los nueve estados de salud son: 1) sano, 2) enfermedad aguda significativa, 3) enfermedad crónica menor única o múltiple, 4) enfermedades crónicas moderadas, 5) enfermedades crónicas dominantes, 6 y 7) múltiples enfermedades crónicas dominantes, 8) enfermedad neoplásica avanzada y 9) enfermedades graves. Cada nivel sucesivo mantiene los niveles de estado 
y de la gravedad, al tiempo que reduce el número de grupos y, en su caso, ajusta los niveles de gravedad.

En síntesis, los CRG son un método muy completo y útil para la estimación de riesgo y el conocimiento epidemiológico de la población, ya que relacionan características históricas, clínicas y demográficas del individuo con cantidad y tipo de recursos sanitarios consumidos (12).

El presente estudio se propone estimar la multimorbilidad asociada con diabetes mellitus tipo 2-DM2- y su relación con el gasto farmacéutico, según el sistema de ajuste de riesgos CRG en la Comunidad Valenciana, España.

\section{Métodos}

\section{Tipo de estudio, población y muestra}

Se trata de un estudio ecológico llevado a cabo en la Comunidad Valenciana (cv), España. La población total residente en dicha comunidad autónoma en el año 2012 fue de 5150054 habitantes, todos ellos dados de alta del Sistema de Información Poblacional (SIP) y disponen de una tarjeta sanitaria con un número de identificación.

De esa población se identificó a 491854 pacientes diagnosticados de DM2. Los criterios de inclusión fueron:

- Pacientes con Clasificación Internacional de Enfermedades- CIE-9-MC 250 y subcategorías, además de los siguientes, por estar relacionados con el uso de tiras reactivas: $648,8,648,0,775,1,790,6$, 790,29, 707,10, 707,9, 731,8, 251,2, 211,7 y 249 y sus subcategorías.
- Pacientes que consumen medicamentos con principios activos antidiabéticos (Sistema de Clasificación Anatómica, Terapéutica, Química -ATC A10: antidiabéticos) y tiras control para la glicemia (ATC V04CA).

- Pacientes clasificados en grupos de riesgo clínico (CRG) en la MDC (major diagnostic category) 101 (diabetes) y/o EDC (episode diagnostic category) 424 (diabetes).

Se excluyó a aquellos pacientes que presentaban diabetes juvenil y los que no recibían tratamiento farmacológico, con lo cual el grupo de estudio se redujo a 350015 personas.

El listado de variables con las que se ha trabajado y que se han analizado en el presente estudio es el siguiente:

Variable principal: gasto farmacéutico anual por paciente en euros.

\section{Variables explicativas}

- Datos sociodemográficos: clave anonimización pacientes, fecha de nacimiento, sexo (variable categórica h/m), edad (como variable numérica discreta), centro de salud, zona, departamento de salud, facultativo asignado, nacionalidad, empadronamiento (categórica: $\mathrm{s} / \mathrm{n}$ ), estado en SIP (activo/pensionista), condición de aportación de farmacia (Tarjeta Sanitaria-TSI-RAF).

- Datos sobre el CRG: número de CRG y sus agrupaciones ACRG1, ACRG2, ACRG3; peso del CRG Canadá EDC 424 (diabetes) episode diagnostic category; EPC (episode procedure category); MDC 101 (diabetes) (major diagnostic category); y $\mathrm{PCD}$ (primary chronic category). 
- Datos de comorbilidades: edc_hipertension_001, edc_dislipemia_445, edc_trastorno_mental_751, edc_alzheimer_6, edc_trastorno_mental_756, edc_enfermedad_osteoarticular_357,edc_ enfermedad_cardiovascular_183, edc_epoc_133, edc_asma_138, edc_obesidad_441, edc_cancer_170, edc_enfermedad_tiroidea_446,edc_diabetes complicaciones_circulatorias_428,edc_ coma_diabetico_429,edc_cetoacidosis_diabetica_430,edc_nefropatia_diabetica_431,edc_neuropatia_diabetica_432,edc_retinopatia_ diabetica_433,edc_otras_complicaciones_diabeticas_434.

- Datos de farmacia relativos a dispensación: principio activo (ATC A10 y ATC v04CA), ATC de nivel 1, ATC de nivel 2, ATC de nivel 3, ATC de nivel 4, ATC de nivel 5, presentación farmacéutica, número de envases, número de formas farmacéuticas, dosis diarias definidas, importe de farmacia ambulatoria en euros, importe de farmacia hospitalaria en euros.

- Datos de temporalidad: año, mes.

\section{Recolección y análisis de la información}

La principal fuente de información del presente estudio fue la Historia Clínica Electrónica (HCE), que se compone de los siguientes módulos: Sistema de Información Poblacional (SIP), dispensación y Sistema de Información Ambulatoria (SIA), así como el Gestor de la Prestación Farmacéutica (GAIA), el Conjunto Mínimo Básico de Datos hospitalarios (CMBD) y el Sistema de Información Económica (SIE).

Por otra parte, para identificar los casos se partió de la base de datos de morbilidad (CRG) poblacional, disponible en la Dirección General de Farmacia de la Consellería de Sanidad de la Comunidad Valenciana, que es el Servicio Regional que Gestiona la Sanidad en esta Comunidad Autónoma.

En cuanto al sistema de agrupación de pacientes CRG, este modelo de agrupación efectúa una primera clasificación, que agrupa los códigos CIE-9-MC en categorías diagnósticas mayores (MDC). Así, del total de pacientes de la Comunidad Valenciana se seleccionaron las 350015 personas que durante el año 2012 fueron clasificadas en la MDC 101 diabetes mellitus, a partir de los códigos CIE-9-MC asignados a sus contactos asistenciales. Cada MDC agrupa diferentes categorías de EDC, construidas también a partir de los códigos CIE-9-MC. Se ha considerado que el EDC 427 diabetes identificaba a los pacientes con DM1 y el EDC 424 diabetes a los pacientes con DM2.

Con los datos obtenidos, se estimaron las frecuencias de ocurrencia de las enfermedades crónicas en la cohorte de pacientes con diabetes tipo 2, seleccionados y clasificados en CRG. Asimismo, se estimó la media anual de gastos de los fármacos y el gasto farmacéutico total en atención primaria, teniendo en cuenta los CRG.

Además, se analizó el consumo de fármacos según el estado de salud de los pacientes. En este punto es importante aclarar que los fármacos antidiabéticos orales (ADO) estudiados se han diferenciado según el mecanismo de acción. Los secretagogos son medicamentos que estimulan a la célula beta para que segregue insulina, incluyen a las sulfonilureas y a las glinidas. El resto de ADO se han considerado no secretagogos.

Los datos empleados fueron analizados utilizando el paquete estadístico Statistical Package for the Social Sciences (SPSs) versión 20.0. El numero sIP de los pacientes (número de identificación) fue remplazado por un número anonimizado e irreversiblemente disociado, con el fin de mantener la confidencialidad de la información. 


\section{Resultados}

La prevalencia de diabetes en la Comunidad Valenciana para el año 2012 se situó en $6,7 \%$, puesto que la población de estudio son 350015 personas. El 7,8\% de la población identificada a través del consumo de fármacos es considerada sana. Del mismo modo, el $85 \%$ de la población diabética identificada a través de los CRG se ubica entre los estado de salud 5 a 7 (ver tabla 1 y figura 1 ).

Casi la mitad de la población diabética $(47,74 \%)$ se encuentra dentro del estado de salud 6 , lo que indica que los pacientes diabéticos se sitúan en estados de salud de elevada morbilidad, y por tanto es lógico que el número de comorbilidades sea elevado en la mayoría de los casos.

Tabla 1. Población diabética de la Comunidad Valencia según el Clinical Risk Group (Crg), 2012

\begin{tabular}{|c|c|c|c|}
\hline CRG & Estado de salud & $\mathbf{N}^{\circ}$ & $\%$ \\
\hline 1 & Sanos & 27300 & 7,8 \\
\hline 2 & Enfermedad aguda significativa & 4722 & 1,3 \\
\hline 3 & Enfermedad crónica menor (única o múltiple) & 9244 & 2,6 \\
\hline 4 & Enfermedades crónicas moderadas & 4352 & 1,2 \\
\hline 5 & Enfermedad crónica dominante & 102365 & 29,2 \\
\hline 6 & Enfermedad crónica importante en múltiples sistemas orgánicos & 167067 & 47,7 \\
\hline 7 & Enfermedad crónica dominante en tres o más sistemas orgánicos & 27779 & 7,9 \\
\hline 8 & Enfermedad neoplásica avanzada & 4991 & 1,4 \\
\hline 9 & Enfermedades graves o catastróficas & 2104 & 0,6 \\
\hline
\end{tabular}

Fuente: Sistema de Información Poblacional (sIP), Dispensación y Sistema de Información Ambulatoria (SIA). Cálculos de los autores, 2012

Figura 1. Porcentaje de pacientes con diabetes tipo 2 en CADA estado de SAlud

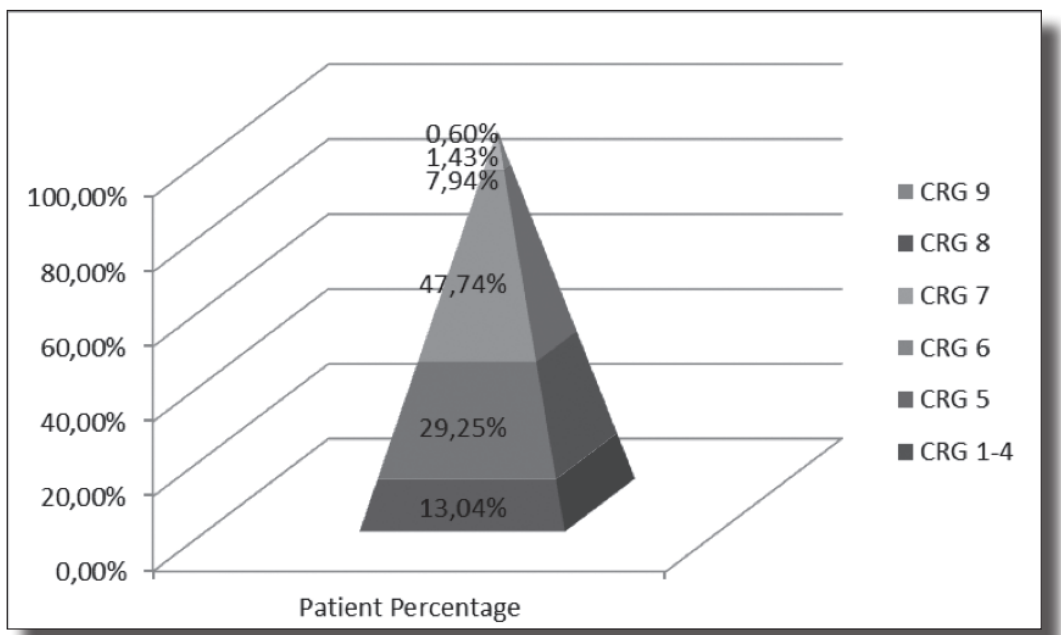

1) sanos 2) enfermedad aguda significativa, 3) enfermedad crónica menor única o múltiple, 4) enfermedades crónicas moderadas, 5) enfermedad crónica dominante, 6) enfermedad crónica importante en múltiples sistemas orgánicos, 7) enfermedad crónica dominante en tres o más sistemas orgánicos, 8) enfermedad neoplásica avanzada, 9) enfermedades graves o catastróficas. 
Luis Alvis-Estrada • David Vivas-Consuelo • Vicent Caballer-Tarazona • Ruth Usó-Talamantes Carla Sancho-Mestre $\bullet$ Laia Buigues-Pastor

Tabla 2. Multimorbilidad en pacientes diabéticos de la Comunidad Valenciana, 2012

\begin{tabular}{|l|c|c|c|c|}
\hline Número de comorbilidades & $\mathbf{N}^{\mathbf{0}}$ & $\%$ & Gasto promedio anual & Desviación estándar \\
\hline Única & 35644 & 10,2 & $€ 592,3$ & $€ 850,1$ \\
\hline Una adicional & 73232 & 20,9 & $€ 887,8$ & $€ 1008,9$ \\
\hline Dos adicionales & 94618 & 27,0 & $€ 1189,6$ & $€ 1076,0$ \\
\hline Tres adicionales & 72610 & 20,7 & $€ 1531,1$ & $€ 1239,8$ \\
\hline Cuatro adicionales & 41505 & 11,9 & $€ 1881,6$ & $€ 1371,0$ \\
\hline Cinco adicionales & 19619 & 5,6 & $€ 2221,5$ & $€ 1532,9$ \\
\hline Seis y más adicionales & 12787 & 3,7 & $€ 3825,41$ & $€ 1802,9$ \\
\hline
\end{tabular}

Fuente: SIP, Dispensación y sIA. Cálculos de los autores, 2012

En concreto, el $90 \%$ (314371) de los diabéticos de la Comunidad Valenciana tienen otra enfermedad además de la diabetes. El $47,7 \%$ (167228) tienen entre dos y tres comorbilidades. El gasto farmacéutico muestra una clara tendencia ascendente, a medida que aumenta el número de comorbilidades.

Los importes de gasto promedio anual por paciente muestran una gran variabilidad en función del número de comorbilidades, en un rango que va de los 592 euros en pacientes con una única comorbilidad hasta los 3825,41 para los pacientes que presentan más de seis comorbilidades adicionales (tabla 2).

Entre las comorbilidades destaca la alta prevalencia de hipertensión, que oscila entre 47 y $97 \%$ en los CRG 5 a 7, así como la dislipemia, que en los mismos CRG oscila entre 50 y $59 \%$. El trastorno mental alcanza su mayor prevalencia en el CRG 7, al igual que la enfermedad cardiovascular (ver tabla 3).

Dado que la mayoría de pacientes se engloban dentro del estado de salud 6, resulta interesante analizar las comorbilidades de los pacientes en este grupo, ya que como ya hemos mencionado, suponen casi el 50\% de la población diabética de la Comunidad Valenciana.
En primer lugar, la hipertensión es la que más afecta a esos pacientes, seguida por la dislipemia, a mucha distancia del resto de comorbilidades (84 y 58\%, respectivamente, sobre la población diabética clasificada en el estado de salud 6). En un segundo grupo encontraríamos comorbilidades que afectan a un $30 \%$, aproximadamente, de los pacientes de este grupo (enfermedad osteorticular y transtorno mental). El resto tienen una incidencia mucho menor y por debajo siempre del $16 \%$ de los pacientes diabéticos en estado de salud 6.

El mayor gasto medio en insulinas se encuentra entre los pacientes del CRG 9. Del mismo modo, el mayor gasto medio en secretagogos y no secretagogos se da en los pacientes de los CRG 5 a 7. En cuanto al gasto medio en tiras reactivas de glucosa en sangre, el mayor rubro fue en el CRG 9. El mayor gasto medio total es de $€ 2840,5$ y corresponde también al CRG 9, como era de esperar.

\section{Discusión y conclusiones}

La utilización de sistemas ajustados al riesgo con el fin de predecir costos en salud, ha sido abordada desde los años noventa, pero en épocas actuales ante la escalada de costos ha cobrado mayor relevancia. La validez de estos 


\begin{tabular}{|c|c|c|c|c|c|c|c|c|c|c|c|c|c|c|}
\hline$d^{\circ}$ & 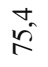 & $\begin{array}{l}\text { Pे } \\
\stackrel{n}{n}\end{array}$ & बें & $\begin{array}{l}0 \\
\stackrel{-}{=}\end{array}$ & $\begin{array}{l}\text { त̂ } \\
\text { ¿̂े }\end{array}$ & $\stackrel{0}{r}$ & $\alpha^{2}$ & $\stackrel{+}{\varrho}$ & $\stackrel{m}{\stackrel{m}{g}}$ & $\stackrel{0}{=}$ & है & $\stackrel{+}{i}$ & $\stackrel{m}{m}$ & $\hat{\sigma}$ \\
\hline $\begin{array}{l}\tilde{z} \\
\tilde{z}\end{array}$ & $\begin{array}{l}\stackrel{D}{\infty} \\
\stackrel{\curvearrowleft}{n}\end{array}$ & $\stackrel{\stackrel{\mathcal{I}}{\exists}}{=}$ & ? & $\underset{n}{\stackrel{R}{n}}$ & 茫 & $\stackrel{\bullet}{\stackrel{-}{-1}}$ & ఫ & $\stackrel{\infty}{\vec{N}}$ & 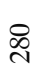 & 志 & ત્ડ & in & g) & $\cong$ \\
\hline$b^{\circ}$ & $\hat{+}$ & $\tilde{q}$ & से & $\overrightarrow{\widetilde{d}}$ & $\stackrel{\text { ? }}{\stackrel{+}{N}}$ & $\stackrel{\circ}{\sim}$ & $\stackrel{n}{2}$ & $\vec{r}$ & $\begin{array}{l}\text { กิ } \\
\text { ते }\end{array}$ & ڤn & $\begin{array}{l}0 \\
\dot{ \pm}\end{array}$ & $\stackrel{\sim}{f}$ & $\stackrel{0}{\oplus}$ & $\Leftrightarrow$ \\
\hline 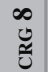 & 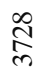 & $\underset{\stackrel{\sim}{\sim}}{\stackrel{\sim}{\sim}}$ & $\frac{\mathcal{I}}{I}$ & $\stackrel{\sqrt{n}}{=}$ & $\stackrel{n}{\beth}$ & $\vec{\infty}$ & 离 & 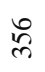 & $\stackrel{ }{\text { 。ㅇ }}$ & চ̂. & $\widehat{N}$ & 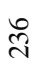 & 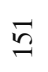 & 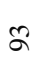 \\
\hline$d^{e}$ & $\overrightarrow{\hat{\sigma}}$ & 夰 & ले & तै & $\frac{n}{f}$ & $\begin{array}{l}\infty \\
\infty\end{array}$ & $\hat{\varrho}$ & $\stackrel{0}{\circ}$ & $\begin{array}{l}\text { थे } \\
\text { ले }\end{array}$ & $\stackrel{\infty}{\sim}$ & $\stackrel{\sim}{2}$ & के & గु & $\hat{\sigma}$ \\
\hline ڤ̆ & 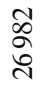 & $\underset{\sim}{\stackrel{d}{d}}$ & 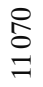 & $\hat{\alpha}$ & $\underset{\sigma}{\sigma}$ & $\underset{\sim}{\stackrel{f}{J}}$ & ఫ్రి & 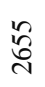 & $\begin{array}{l}\text { do } \\
\stackrel{2}{0}\end{array}$ & $\frac{\stackrel{n}{\sim}}{\sim}$ & $\underset{n}{\tilde{n}}$ & 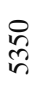 & $\stackrel{ }{\Xi}$ & $\hat{\approx}$ \\
\hline de & $\begin{array}{l}m \\
\stackrel{\infty}{\infty}\end{array}$ & $\overrightarrow{\hat{\infty}^{\circ}}$ & $\hat{\hat{े}}$ & $\stackrel{+}{\stackrel{n}{n}}$ & $\stackrel{0}{0}$ & $\hat{\infty}$ & $\stackrel{\forall}{\sigma}$ & $\stackrel{t}{\varrho}$ & है & $\stackrel{0}{\sim}$ & $\ddot{\theta}_{0}$ & ले & $F$ & 3 \\
\hline $\begin{array}{l}\text { b } \\
\text { ֶ̋ }\end{array}$ & $\begin{array}{l}\infty \\
\stackrel{\infty}{\circ} \\
\stackrel{+}{+}\end{array}$ & 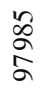 & $\begin{array}{l}\text { 苨 } \\
\stackrel{q}{q}\end{array}$ & $\begin{array}{l}8 \\
\infty \\
\infty \\
i n\end{array}$ & 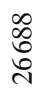 & 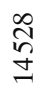 & $\begin{array}{l}\text { J } \\
\text { 号 }\end{array}$ & $\begin{array}{l}\stackrel{2}{ } \\
\stackrel{2}{=}\end{array}$ & $\stackrel{\infty}{\underset{0}{\infty}}$ & 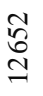 & $\stackrel{+}{\stackrel{0}{0}}$ & బ & $\tilde{\sigma}$ & $\underset{+}{\stackrel{\infty}{+}}$ \\
\hline$\therefore$ & $\begin{array}{l}\approx \\
\stackrel{\gamma}{\gamma}\end{array}$ & $\overrightarrow{\hat{n}}$ & $\stackrel{\circ}{\circ} \stackrel{0}{\infty}$ & $\begin{array}{l}n \\
\infty \\
\infty\end{array}$ & $\stackrel{\sim}{r}$ & $\vec{r}$ & हो & $\stackrel{0}{+}$ & $\stackrel{m}{i}$ & $\stackrel{3}{i}$ & $\stackrel{\sim}{\infty}$ & $\stackrel{6}{\rightarrow}$ & $\stackrel{0}{-}$ & $\overrightarrow{0}$ \\
\hline 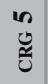 & $\begin{array}{l}\text { જે } \\
\text { के }\end{array}$ & $\begin{array}{l}\text { ô } \\
\stackrel{\sim}{n} \\
\text { nd }\end{array}$ & 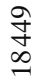 & 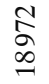 & $\stackrel{n}{\underset{7}{f}}$ & $\underset{\sim}{\stackrel{N}{N}}$ & ठ̊ర్రి & 令 & 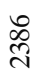 & 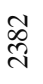 & तે & $\underset{\sim}{\widetilde{0}}$ & 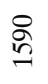 & $\underset{\sigma}{\stackrel{d}{\sigma}}$ \\
\hline$\therefore$ & $\begin{array}{l}\infty \\
\infty \\
\infty \\
m\end{array}$ & $\hat{m}$ & $\begin{array}{l}\stackrel{0}{2} \\
\stackrel{2}{\Omega}\end{array}$ & $\stackrel{?}{\sim}$ & $\stackrel{\sim}{\sim}$ & $\overbrace{0}^{+}$ & $\stackrel{+}{+}$ & $\hat{i}$ & $\stackrel{0}{-}$ & $\stackrel{n}{\rightarrow}$ & $\stackrel{+}{i}$ & $\stackrel{0}{-}$ & $\stackrel{+}{\rightarrow}$ & $\overrightarrow{0}$ \\
\hline $\begin{array}{l}\frac{\pi}{\pi} \\
\frac{\tilde{g}}{\tilde{U}}\end{array}$ & 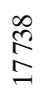 & 字 & 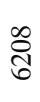 & $\frac{0}{\stackrel{0}{\circ}}$ & 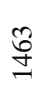 & ন & ڤ્ণ & ్ָ & $\cong$ & $\begin{array}{l}\infty \\
\infty\end{array}$ & $\begin{array}{l}\infty \\
\stackrel{\infty}{0}\end{array}$ & 导 & $\bar{b}$ & $m$ \\
\hline 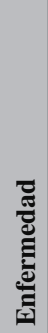 & 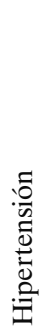 & 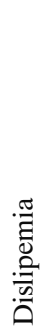 & 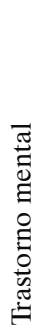 & 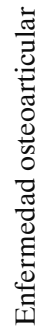 & 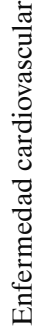 & 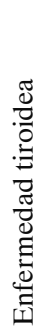 & 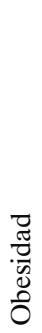 & $\begin{array}{l}\frac{n}{n} \\
0 \\
0 \\
0 \\
0 \\
0 \\
0 \\
0 \\
0\end{array}$ & $\begin{array}{l}\text { O } \\
\text { Oे } \\
\text { in }\end{array}$ & 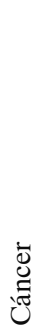 & 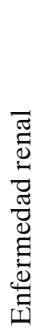 & $\begin{array}{l}\overrightarrow{\mathrm{d}} \\
\text {. } \\
\frac{\bar{N}}{\mathrm{~N}}\end{array}$ & 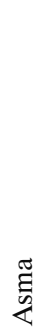 & 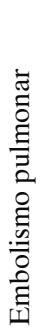 \\
\hline
\end{tabular}


Figura 2. Número de PaCientes POR COMORbILIDAd EN El ESTAdo de SALUd 6 del CRG

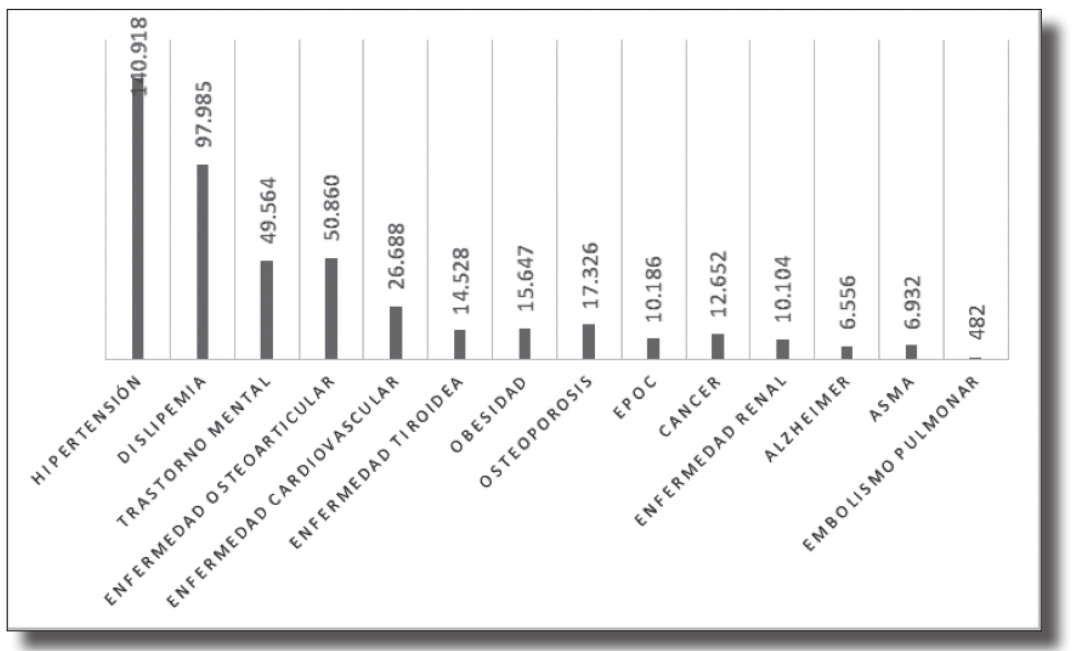

Fuente: elaboración propia

Tabla 4. Gasto medio en fármacos SEGún CRG, 2012

\begin{tabular}{|l|c|c|c|c|c|}
\hline \multirow{2}{*}{} & \multicolumn{5}{|c|}{ Gasto medio en fármacos } \\
\cline { 2 - 6 } & CRG 1 A 4 & CRG 5 A 7 & CRG 8 & CRG 9 & Total \\
\hline Insulinas & $€ 33,1$ & $€ 128,6$ & $€ 147,3$ & $€ 248,7$ & $€ \mathbf{1 1 7 , 1}$ \\
\hline $\begin{array}{l}\text { ADO } \\
\text { secretagogos }\end{array}$ & $€ 8,8$ & $€ 16,3$ & $€ 12,6$ & $€ 6,2$ & $\mathbf{€ 1 5 , 2}$ \\
\hline $\begin{array}{l}\text { ADO } \\
\text { No secretagogos }\end{array}$ & $€ 95,7$ & $€ 232,8$ & $€ 154,9$ & $€ 87,0$ & $\mathbf{€ 2 1 2 , 9}$ \\
\hline Tiras & $€ 119,5$ & $€ 209,0$ & $€ 213,3$ & $€ 283,0$ & $\mathbf{€ 2 0 2 , 3}$ \\
\hline Gasto total & $€ 476,5$ & $€ 1350,5$ & $€ 2174,1$ & $€ 2840,5$ & $\mathbf{€ 1 2 5 7 , 1}$ \\
\hline
\end{tabular}

Fuente: SIP, Dispensación y sia. Cálculos de los autores, 2012

sistemas ha sido sustentada. Por ejemplo, Hughes estableció 269 CRG basados en la presencia de enfermedades crónicas y en combinaciones de este tipo de enfermedades, teniendo en cuenta el historial clínico en afiliados a Medicare y Medicaid. Estos CRG se agruparon en las nueve categorías asumidas en el presente estudio y mostraron tener una alta concordancia en el gasto causado y el gasto predicho (9).

Lo anterior facilita el análisis financiero de la gestión de medicamentos y clínica en general, que suele ser cada vez más común en los sistemas de salud en el mundo (13). Todo esto descansa sobre la base de un sistema de clasificación de pacientes que en algunos casos, como en el de los CRG, se encuentra basado en el consumo de medicamentos o fármacos (14). El establecimiento de un sistema de clasificación de pacientes permite gestionar de mejor manera las enfermedades crónicas, predecir el consumo de recursos y determinar perfiles de consumo farmacéutico.

En este estudio, la prevalencia de DM2 hallada es menor que la reportada por Catalá (3) para la Comunidad Valenciana, la cual fue de $10,8 \%$, según los parámetros de la OMs, y 
similar a otras regiones de España. Asimismo, en la población diabética de la Comunidad Valenciana se destaca la alta prevalencia de comorbilidades, lo cual resulta similar a lo reportado por otros estudios en poblaciones similares (15-17).

Como cabría esperar, la comorbilidad más frecuente resultó ser la hipertensión. La prevalencia de esta comorbilidad concuerda con lo reportado a nivel mundial en la población diabética (18).

Por otro lado, el gasto medio estimado en la población diabética de la Comunidad Valenciana es similar al reportado a nivel mundial, el cual equivale a USD 1522 (19), mostrando con ello la precisión en la obtención de la estimación de gastos que tiene el análisis basado en CRG. Asimismo, el gasto medio encontrado supone un promedio mensual de $€ 104,7$, que representan alrededor del $14 \%$ del salario mínimo de España.

$\mathrm{Al}$ agruparse el $85 \%$ de los pacientes diabéticos en los estados de salud CRG 5 a 7, queda claro que estos grupos son quienes mayormente representan a este perfil de pacientes. En estudios previos se ha reportado que solo los pacientes dentro de los grupos CRG $5 \mathrm{y}$ 6 representan el gasto total farmacéutico causado en la Comunidad Valenciana (20).

Por último, a pesar de las bondades ofrecidas por un sistema de ajuste basado en la clasificación de pacientes como el resaltado en el presente estudio, el establecimiento de dicho sistemas en nuestro país debe considerar las condiciones propias del sistema de salud, que son distintas al contexto europeo y estadounidense.

\section{Referencias bibliográficas}

1. International Diabetes Federation. The IDF Diabetes Atlas. Bruselas: International Diabetes Federation; 2011.
2. Soriguer F, Goday A, Bosch-Comas A, Bordiu E, Calle-Pascual A, Carmena R, et al. Prevalence of diabetes mellitus and impaired glucose regulation in Spain: the Di@bet.es Study. Diabetologia. 2012; 55 (1): 88-93.

3. Catalá M, Girbes J, Bataller A, Lluch I, Dolz A, Pedro T, et al. Estudio Valencia: investigación de la prevalencia de diabetes mellitus y síndrome metabólico. Generalitat Valenciana. Consellería de Sanitat; 2010.

4. Organización Mundial de la Salud. Diabetes. Nota descriptiva $n^{\circ} 312 ; 2011$.

5. Crespo C, Brosa M, Soria A, López A, López N, Soria B. Costes directos de la diabetes mellitus y de sus complicaciones en España (Estudio SECCAID: Spain estimated cost Ciberdem-Cabimer in Diabetes). Avances en Diabetología. 2013; 30 (5): 182-9.

6. Liebl A, Neiss A, Spannheimer A, Reitberger U, Wieseler B, Stammer H, et al. Complications, co-morbidity, and blood glucose control in type 2 diabetes mellitus patients in Germany--results from the CODE-2 study. Experimental and Clinical Endocrinology \& Diabetes: Official Journal, German Society of Endocrinology [and] German Diabetes Association. 2002; 110 (1):10-6.

7. Ellis RP, Pope GC, Iezzoni L, Ayanian JZ, Bates DW, Burstin H, et al. Diagnosis-based risk adjustment for Medicare capitation payments. Health Care Financing Review. 1996; 17 (3): 101-28.

8. Starfield B, Weiner J, Mumford L, Steinwachs D. Ambulatory care groups: a categorization of diagnoses for research and management. Health Services Research. 1991; 26 (1): 53-74.

9. Hughes JS, Averill RF, Eisenhandler J, Goldfield NI, Muldoon J, Neff JM, et al. Clinical Risk Groups (CRGS): a classification system for risk-adjusted capitation-based payment and health care management. Medical Care. 2004; 42 (1): 81-90.

10. Vivas D, Guadalajara N, Barrachina I, Trillo JL, Uso R, de-la-Poza E. Explaining primary healthcare pharmacy expenditure using classification of medications for chronic conditions. Health Policy. 2011; 103 (1): 9-15.

11. Vivas D, Usó R, Trillo J, Caballer M, Barrachina I, Buigues L. Predictability of pharmaceutical spending in primary health services using Clinical Risk Groups. Health Policy. 2014; 11 (2-3): 188-95.

12. Romero M, Alturo A, Garzón C, Arango C, Romero D, Jiménez I, et al. Herramienta metodológica para la toma de decisiones. Cartilla del modelo del monitoreo. Bogotá: Escuela Superior de Administración Pública (ESAP); 2014.

13. Caballer M, Buigues L, Saurí I, Uso R, Trillo J. Analysis and assessment of models of pharmaceutical expenditure prediction. [A Standardized Amount Indicator by Equivalent Patient to Control Outpatient Pharmaceutical Expenditure, Spain]. Revista Española de Salud Pública. 2011; 86: $371-80$

14. Von Korff M, Wagner E, Saunders K. Chronic disease score from automated pharmacy data. Journal of Clinical Epidemiology. 1992; 45: 197-203. 
15. Almawi W, Tamim H, Al-Sayed N, Arekat MR, Al-Khateeb GM, Baqer A, et al. Association of comorbid depression, anxiety, and stress disorders with Type 2 diabetes in Bahrain, a country with a very high prevalence of Type 2 diabetes. Journal of Endocrinological Investigation. 2008; 31 (11): 1020-4.

16. Kilzieh N, Rastam S, Maziak W, Ward KD. Comorbidity of depression with chronic diseases: a population-based study in Aleppo, Syria. International Journal of Psychiatry in Medicine. 2008; 38 (2): 169-84.

17. wHO. Rio Political Declaration on Social Determinants of Health. The World Conference on
Social Determinants of Health; Río de Janeiro, Brasil, 2011.

18. Arauz-Pacheco C, Parrott MA, Raskin P. The treatment of hypertension in adult patients with diabetes. Diabetes Care. 2002; 25 (1): 134-47.

19. Federación Internacional de Diabetes. Atlas de la diabetes de la FID. Federación Internacional de Diabetes; 2014.

20. Vivas D, Usó R, Guadalajara N, Trillo J, Sancho C, Buigues L. Pharmaceutical cost management in an ambulatory setting using a risk adjustment tool. BMC Health Services Research. 2014; 14: 462. 